\title{
Joint IterativeTx/Rx MMSE-FDE and ISI Cancellation for Single-Carrier Hybrid ARQ with Chase Combining
}

\author{
Kazuki Takeda and Fumiyuki Adachi \\ Department of Electrical and Communication Engineering, Graduate School of Engineering, Tohoku University, 6-6-05, Aza-Aoba, \\ Aramaki, Aoba-ku, Sendai 980-8579, Japan \\ Correspondence should be addressed to Kazuki Takeda, kazuki@mobile.ecei.tohoku.ac.jp
}

Received 15 April 2010; Revised 25 July 2010; Accepted 2 September 2010

Academic Editor: D. D. Falconer

Copyright (๑) 2011 K. Takeda and F. Adachi. This is an open access article distributed under the Creative Commons Attribution License, which permits unrestricted use, distribution, and reproduction in any medium, provided the original work is properly cited.

\begin{abstract}
We propose a joint iterative transmit/receive ( $\mathrm{Tx} / \mathrm{Rx}$ ) minimum mean square error (MMSE) frequency-domain equalization (FDE) and intersymbol interference cancellation (ISIC) suitable for single-carrier (SC) hybrid automatic repeat request (HARQ) with chase combining (CC). In the proposed scheme, a one-tap transmit MMSE-FDE and an iterative one-tap receive MMSE-FDE and ISIC are employed. Every time the retransmitted packet is received, a series of receive MMSE-FDE, antenna diversity combining, packet combining, and ISIC is carried out in an iterative manner by using updated receive MMSE-FDE weights in each iteration. The transmit MMSE-FDE weight is determined based on the prediction of the degree of residual ISI after the iteration process in a receiver. We theoretically derive a set of transmit and receive MMSE-FDE weights. We show by computer simulation that joint iterative Tx/Rx MMSE-FDE and ISIC achieves better packet error rate (PER) and higher throughput of SC-HARQ than the conventional Rx MMSE-FDE and ISIC.
\end{abstract}

\section{Introduction}

The broadband wireless channel comprises many propagation paths having different time delays [1]. The minimum mean square error frequency-domain equalization (MMSEFDE) provides good bit error rate (BER) performance for broadband single-carrier (SC) transmissions in a severe frequency-selective fading channel [2-4]. However, the performance of SC using the MMSE-FDE is still a few $\mathrm{dB}$ away from the theoretical lower-bound due to residual intersymbol interference (ISI) after MMSE-FDE. The use of iterative MMSE-FDE and ISI cancellation (ISIC) has been extensively studied [5-10]. In [5-8], iterative receive (Rx) MMSE-FDE and ISIC was proposed. The receive FDE weight is updated based on the MMSE criterion by using the reliability information of data detection at the previous iteration. In [8], channel decoding is incorporated into each iteration process to further improve the performance. In $[9,10]$, iterative Rx MMSE-FDE and ISIC was proposed for SC-hybrid automatic repeat request (HARQ).
Recently, we took a different approach from the iterative receive processing and proposed a joint transmit/receive $(\mathrm{Tx} / \mathrm{Rx})$ MMSE-FDE [11] that exploits the channel state information (CSI) at both the transmitter and the receiver to improve the performance of broadband SC transmissions. In [11], a set of transmit and receive MMSE-FDE weights was derived based on the MMSE criterion. It was shown [11] that the proposed scheme achieves better BER performance than the conventional receive MMSE-FDE. More recently, we proposed joint iterative $\mathrm{Tx} / \mathrm{Rx}$ MMSE-FDE and ISIC that is an extension of joint $\mathrm{Tx} / \mathrm{Rx}$ MMSE-FDE [12]. In this scheme, iterative Rx MMSE-FDE and ISIC proposed in [5-8] is incorporated into joint $\mathrm{Tx} / \mathrm{Rx}$ MMSE-FDE. At the transmitter, one-tap transmit MMSE-FDE is carried out using CSI before transmitting the signal. At the receiver, by viewing a concatenation of transmit MMSE-FDE and the channel as a new equivalent channel, one-tap receive MMSEFDE and ISIC is carried out in an iterative manner similar to [5-8]. In each iteration stage, the receive MMSE-FDE weight is updated so as to reduce the residual ISI significantly. 
On the other hand, the transmit MMSE-FDE weight is computed based on the prediction of the degree of residual ISI after ISIC at the receiver. Joint iterative Tx/Rx MMSEFDE and ISIC provides much better BER performance than the conventional iterative MMSE-FDE and ISIC.

In this paper, we propose a joint iterative $\mathrm{Tx} / \mathrm{Rx}$ MMSEFDE and ISIC suitable for SC-HARQ with Chase combining (CC) [13-15]. In CC, the same packet is retransmitted until it is correctly received. In the proposed scheme, a onetap transmit MMSE-FDE and an iterative one-tap receive MMSE-FDE and ISIC are employed. Every time the retransmitted packet is received, a series of receive MMSE-FDE, antenna diversity combining, packet combining, and ISIC is carried out in an iterative manner by using receive MMSEFDE weights updated in each iteration. In the proposed scheme, the transmitter predicts the residual ISI power after the receiver performs the iterative Rx MMSE-FDE and ISIC. Based on the predicted residual ISI power, the transmit MMSE-FDE weight is determined. We evaluate by computer simulation the packet error rate (PER) and throughput performances achievable by the proposed scheme and compare them with those achievable by the conventional scheme proposed in $[9,10]$.

In this paper, we assume perfect knowledge of CSI. However, in practical systems, CSI should be estimated to compute the transmit and receive MMSE-FDE weights. Assuming the time-division duplex (TDD) systems, where the same carrier frequency is used for the transmission and reception and using the channel reciprocity [16], the transmitter can estimate the CSI of the transmitting channel by using the received signal and compute the transmit MMSE-FDE weight. In [17], we evaluated the impact of imperfect CSI on the performance of the joint Tx/Rx MMSEFDE (ISIC and packet combining were not introduced in [17]). Assuming the TDD systems, it was shown that when practical time-multiplexed pilot-assisted channel estimation is used, the joint Tx/Rx MMSE-FDE always provides better performance than the Rx MMSE-FDE except for a very high mobility situation (e.g., several hundreds $\mathrm{km} / \mathrm{h}$ assuming $100 \mathrm{MHz}$ signal bandwidth at the carrier frequency $5 \mathrm{GHz}$ ). A detailed discussion on the impact of imperfect CSI for the joint iterative Tx/Rx MMSE-FDE and ISIC is left as an important future study.

The rest of this paper is organized as follows. Section 2 describes the system model of SC-HARQ using joint iterative $\mathrm{Tx} / \mathrm{Rx}$ MMSE-FDE and ISIC. In Section 3, a set of transmit and receive MMSE-FDE weights is derived. Section 4 shows the computer simulation results. Section 5 concludes the paper.

\section{SC-HARQ Using Joint Iterative Tx/Rx MMSE-FDE and ISIC}

Figure 1 illustrates a conceptual diagram of SC-HARQ using joint iterative $\mathrm{Tx} / \mathrm{Rx}$ MMSE-FDE and ISIC. The transmitter/receiver structure is illustrated in Figure 2. $N_{r}$ receive antennas are assumed for diversity reception at the receiver [18]. In case of the CC strategy, if any error is detected, the same packet is retransmitted. After receiving the retransmitted packet, the receive MMSE-FDE weight is updated for diversity combining and packet combining. Diversity combining and packet combining using MMSEFDE and ISIC are jointly carried out in an iterative manner. If no error is detected, the ACK signal is sent to request the transmission of a new packet.

The difference between the proposed scheme and the conventional scheme proposed in $[9,10]$ is the introduction of transmit FDE. Since the transmit FDE is a simple one-tap FDE (which consists of fast Fourier transform (FFT), weight multiplication on each frequency, and inverse FFT (IFFT)), the computational complexity is not significantly increased compared to the conventional scheme.

Below, symbol-spaced discrete-time signal representation is used. The $(M-1)$ th packet retransmission is considered, that is, $M$ copies of the same packet have been received. Perfect knowledge of CSI is assumed.

2.1. Transmit Signal. The packet consists of blocks of $N_{c}$ symbols each, where $N_{c}$ is the size of FFT and IFFT. Without loss of generality, we consider the transmission of one $N_{c^{-}}$ symbol block in a packet.

The $N_{c}$-symbol block is represented as $\mathbf{d}=[d(0), \ldots$, $\left.d(t), \ldots, d\left(N_{c}-1\right)\right]^{T}$, where $(\cdot)^{T}$ denotes the transpose operation. $N_{c}$-point FFT is applied to $\mathrm{d}$ to obtain the frequency-domain transmit signal $\mathbf{D}=[D(0), \ldots$, $\left.D(k), \ldots, D\left(N_{c}-1\right)\right]^{T}$ as

$$
\mathrm{D}=\mathbf{F} \mathbf{d}
$$

where

$$
\begin{aligned}
& \mathbf{F}=\frac{1}{\sqrt{N_{c}}} \text {. } \\
& \times\left[\begin{array}{cccc}
1 & 1 & \cdots & 1 \\
1 & e^{-j 2 \pi(1 \times 1) / N_{c}} & \cdots & e^{-j 2 \pi\left(1 \times\left(N_{c}-1\right)\right) / N_{c}} \\
\vdots & \vdots & \ddots & \vdots \\
1 & e^{-j 2 \pi\left(\left(N_{c}-1\right) \times 1\right) / N_{c}} & \cdots & e^{-j 2 \pi\left(\left(N_{c}-1\right) \times\left(N_{c}-1\right)\right) / N_{c}}
\end{array}\right]
\end{aligned}
$$

is an $N_{c} \times N_{c}$ unitary discrete Fourier transform (DFT) matrix.

The transmit FDE weight multiplied to the $k$ th frequency component $D(k)$ of a block in the $(M-1)$ th retransmitting packet is denoted by $W_{t}^{(M-1)}(k), k=0 \sim N_{c}-1$. Before the $(M-1)$ th retransmission, the transmit MMSE-FDE is carried out as

$$
\begin{aligned}
\mathbf{S}^{(M-1)} & =\left[S^{(M-1)}(0), \ldots, S^{(M-1)}(k), \ldots, S^{(M-1)}\left(N_{c}-1\right)\right]^{T} \\
& =\mathbf{W}_{t}^{(M-1)} \mathbf{D},
\end{aligned}
$$

where $\mathbf{W}_{t}^{(M-1)}=\operatorname{diag}\left\{W_{t}^{(M-1)}(0), \ldots, W_{t}^{(M-1)}(k), \ldots, W_{t}^{(M-1)}\right.$ $\left.\times\left(N_{c}-1\right)\right\}$ is an $N_{c} \times N_{c}$ diagonal transmit MMSE-FDE weight matrix for the $(M-1)$ th retransmission under the transmit power constraint $\operatorname{tr}\left[\mathbf{W}_{t}^{(M-1)} \mathbf{W}_{t}^{(M-1) H}\right]=N_{c}$ to 


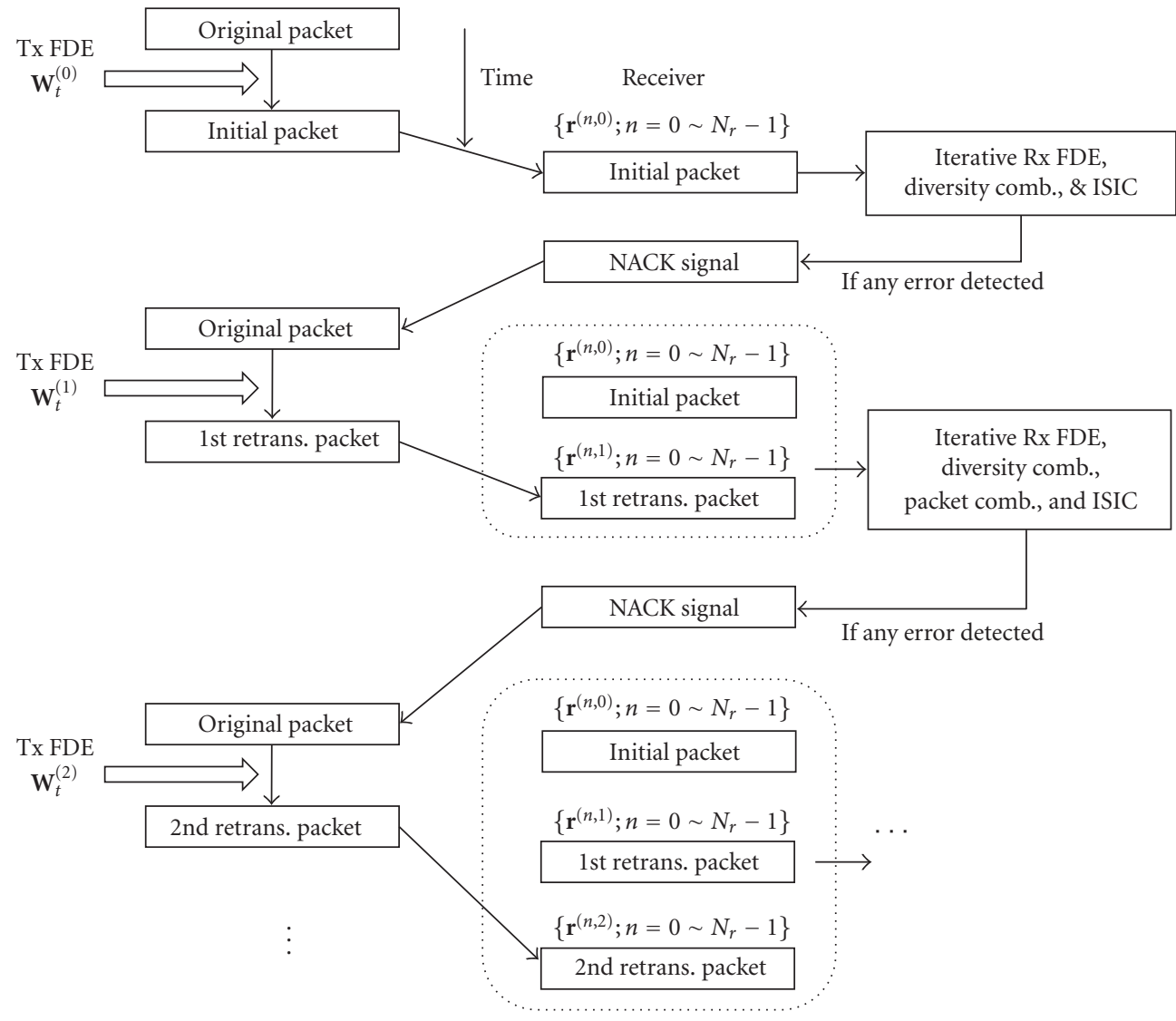

FIgURE 1: SC-HARQ using joint iterative Tx/Rx MMSE-FDE and ISIC.

keep the transmit power intact, where $\operatorname{diag}\{\cdot\}$ denotes the diagonal matrix whose $(k, k)$ th element is given by the $k$ th element of the input vector and $(\cdot)^{H}$ denotes the Hermitian transpose operation.

An $N_{c}$-point IFFT is applied to $\mathbf{S}^{(M-1)}$ to obtain the signal block $\boldsymbol{s}^{(M-1)}=\left[s^{(M-1)}(0), \ldots, s^{(M-1)}(t), \ldots\right.$, $\left.s^{(M-1)}\left(N_{c}-1\right)\right]^{T}=\mathbf{F}^{H} \mathbf{S}^{(M-1)}$. After the insertion of $N_{g^{-}}$ sample cyclic prefix (CP) into the guard interval (GI), the signal block is transmitted.

2.2. Received Signal. The propagation channel is assumed to be an $L$-path frequency-selective block fading channel. The complex-valued path gain and time delay of the $l$ th path between the transmit antenna and $n$th receive antenna for the $m$ th retransmission are, respectively, denoted by $h_{l}{ }^{(n, m)}$ and $\tau_{l}^{(n, m)}, l=0 \sim L-1, n=0 \sim N_{r}-1, m=0 \sim M-1$, with $\sum_{l=0}^{L-1} E\left[\left|h_{l}^{(n, m)}\right|^{2}\right]=1(E[\cdot]$ denotes the ensemble average operation). The CP-length is assumed to be equal to or longer than the maximum channel time delay $\tau_{L-1}$. The received signal block $\mathbf{r}^{(n, m)}=\left[r^{(n, m)}(0), \ldots, r^{(n, m)}(t), \ldots, r^{(n, m)}\left(N_{c}-1\right)\right]^{T}$ on the $n$th receive antenna can be expressed, after the CPremoval, as

$$
\mathbf{r}^{(n, m)}=\sqrt{\frac{2 E_{s}}{T_{s}}} \mathbf{h}^{(n, m)} \mathbf{s}^{(m)}+\mathbf{n}^{(n, m)},
$$

where $E_{s}$ and $T_{s}$ are, respectively, the average transmit symbol energy and symbol duration, $\mathbf{h}^{(n, m)}$ is an $N_{c} \times N_{c}$ circulant channel matrix given by

$$
\mathbf{h}^{(n, m)}=\left[\begin{array}{cccccc}
h_{0}^{(n, m)} & & & h_{L-1}^{(n, m)} & \cdots & h_{1}^{(n, m)} \\
h_{1}^{(n, m)} & \ddots & & & \ddots & \vdots \\
\vdots & & h_{0}^{(n, m)} & \mathbf{0} & & h_{L-1}^{(n, m)} \\
h_{L-1}^{(n, m)} & & h_{1}^{(n, m)} & \ddots & & \\
& \ddots & \vdots & & \ddots & \\
\mathbf{0} & & h_{L-1}^{(n, m)} & \cdots & \cdots & h_{0}^{(n, m)}
\end{array}\right],
$$

and $\mathbf{n}^{(n, m)}=\left[n^{(n, m)}(0), \ldots, n^{(n, m)}(t), \ldots, n^{(n, m)}\left(N_{c}-1\right)\right]^{T}$ is the noise vector with $n^{(n, m)}(t)$ being a zero-mean additive white Gaussian noise (AWGN) having variance $2 N_{0} / T_{s}\left(N_{0}\right.$ is the one-sided noise power spectrum density).

An $N_{c}$-point FFT is carried out on $\mathbf{r}^{(n, m)}$ to obtain the frequency-domain received signal $\mathbf{R}^{(n, m)}$ as

$$
\mathbf{R}^{(n, m)}=\mathbf{F} \mathbf{r}^{(n, m)}=\sqrt{\frac{2 E_{s}}{T_{s}}} \mathbf{H}^{(n, m)} \mathbf{W}_{t}^{(m)} \mathbf{D}+\mathbf{N}^{(n, m)},
$$

where $\mathbf{N}^{(n, m)}=\mathbf{F} \mathbf{n}^{(n, m)}$ and $\mathbf{H}^{(n, m)}=\mathbf{F h}^{(n, m)} \mathbf{F}^{H}$. Due to the circulant property of $\mathbf{h}^{(n, m)}$, the channel gain matrix $\mathbf{H}^{(n, m)}$ of 
size $N_{c} \times N_{c}$ is diagonal. The $k$ th diagonal element of $\mathbf{H}^{(n, m)}$ is given by

$$
H^{(n, m)}(k)=\sum_{l=0}^{L-1} h_{l}^{(n, m)} \exp \left(-j 2 \pi k \frac{\tau_{l}^{(n, m)}}{N_{c}}\right) .
$$

2.3. Joint Iterative Rx MMSE-FDE, Antenna Diversity, Packet Combining, and ISIC. At the receiver, MMSE-FDE, antenna diversity combining, packet combining, and ISIC are jointly carried out in each iteration stage. The maximum number of iterations is denoted by $I$. Below, the $i$ th iteration stage $(0<$ $i \leq I)$ is described.

$\mathbf{R}^{(n, m)}$ is multiplied by the receive MMSE-FDE weight $\mathbf{W} r^{(n, m, i)}=\operatorname{diag}\left\{W_{r}^{(n, m, i)}(0), \ldots, W_{r}^{(n, m, i)}(k), \ldots, W_{r}^{(n, m, i)}\left(N_{c}-\right.\right.$ $1)\}$ before packet combining. The residual ISI replica is generated using the decision result at the $(i-1)$ th iteration stage and is subtracted from the frequency-domain signal after packet combining to obtain $\widehat{\mathbf{D}}^{(i)}=\left[\hat{D}^{(i)}(0), \ldots, \hat{D}^{(i)}(k)\right.$, $\left.\ldots, \hat{D}^{(i)}\left(N_{c}-1\right)\right]^{T}$ as

$$
\widehat{\mathbf{D}}^{(i)}=\sum_{n=0}^{N_{r}-1} \sum_{m=0}^{M-1} \mathbf{W}_{r}^{(n, m, i)} \mathbf{R}^{(n, m)}-\mathbf{\Theta}^{(i-1)},
$$

where

$$
\boldsymbol{\Theta}^{(i-1)}=\sqrt{\frac{2 E_{s}}{T_{s}}}\left\{\sum_{n=0}^{N_{r}-1} \sum_{m=0}^{M-1} \mathbf{W}_{r}^{(n, m, i)} \mathbf{H}^{(n, m)} \mathbf{W}_{t}^{(m)}-\mathbf{I}\right\} \tilde{\mathbf{D}}^{(i-1)}
$$

is the frequency-domain residual ISI replica. In (10), $\widetilde{\mathbf{D}}^{(i-1)}$ is the frequency-domain soft symbol replica given as

$$
\widetilde{\mathbf{D}}^{(i-1)}=\mathbf{F} \tilde{\mathbf{d}}^{(i-1)}
$$

where $\tilde{\mathbf{d}}^{(i-1)}=\left[\tilde{d}^{(i-1)}(0), \ldots, \tilde{d}^{(i-1)}(t), \ldots, \tilde{d}^{(i-1)}\left(N_{c}-1\right)\right]^{T}$ is the soft symbol replica block. The $t$ th element $\tilde{d}^{(i-1)}(t)$ of $\tilde{\mathbf{d}}^{(i-1)}$ is given as [5-10]

$$
\begin{aligned}
& \tilde{d}^{(i-1)}(t) \\
& =\left\{\begin{array}{c}
\frac{1}{\sqrt{2}}\left\{\tanh \left(\frac{\lambda_{t}^{(i-1)}(0)}{2}\right)+j \tanh \left(\frac{\lambda_{t}^{(i-1)}(1)}{2}\right)\right\} \\
\frac{1}{\sqrt{10}}\left\{\tanh \left(\frac{\lambda_{t}^{(i-1)}(0)}{2}\right)\left(2+\tanh \left(\frac{\lambda_{t}^{(i-1)}(1)}{2}\right)\right)\right\} \\
+\frac{j}{\sqrt{10}}\left\{\tanh \left(\frac{\lambda_{t}^{(i-1)}(2)}{2}\right)\left(2+\tanh \left(\frac{\lambda_{t}^{(i-1)}(3)}{2}\right)\right)\right\} \\
\text { for } 16 \text { QAM, }
\end{array}\right.
\end{aligned}
$$

where $\lambda_{t}^{(i-1)}(x)$ is the log-likelihood ratio (LLR) associated with the $x$ th bit of the $t$ th symbol in a block, computed at the $(i-1)$ th iteration stage (note that $x=0 \sim \log _{2} X-1$ and $t=0 \sim N_{c}-1$ ( $X$ is the modulation level) and $\tilde{\mathbf{d}}^{(0)}=\mathbf{0}$ for the first iteration stage).
$\widehat{\mathbf{D}}^{(i)}$ in (8) is transformed into a time-domain signal block by IFFT. After receiving all the transmitted blocks in a packet, channel decoding is applied. The channel decoder output LLR is used to generate the residual ISI replica to be used in the next iteration stage.

\section{Derivation of TX and RX MMSE-FDE Weights}

The MMSE-based transmit and receive FDE weights are derived assuming that $\mathrm{M}$ copies of the same packet have been received. First, we derive the receive MMSE-FDE weights for the given transmit FDE weight. Then, the transmit MMSEFDE weight is derived assuming that the predicted receive MMSE-FDE weight is used.

3.1. Receive MMSE-FDE Weight. A concatenation of the transmit FDE and the propagation channel is viewed as an equivalent channel. We introduce an expanded received signal vector $\mathbf{R}$ of size $M N_{r} N_{c} \times 1$ defined as

$$
\mathbf{R}=\left[\begin{array}{c}
\mathbf{R}^{(0,0)} \\
\vdots \\
\mathbf{R}^{(0, M-1)} \\
\vdots \\
\vdots \\
\mathbf{R}^{\left(N_{r}-1,0\right)} \\
\vdots \\
\mathbf{R}^{\left(N_{r}-1, M-1\right)}
\end{array}\right]=\sqrt{\frac{2 E_{s}}{T_{s}}} \overline{\mathbf{H}} \mathbf{D}+\mathbf{N}
$$

where

$$
\overline{\mathbf{H}}=\left[\begin{array}{c}
\mathbf{H}^{(0,0)} \mathbf{W}_{t}^{(0)} \\
\vdots \\
\mathbf{H}^{(0, M-1)} \mathbf{W}_{t}^{(M-1)} \\
\vdots \\
\vdots \\
\mathbf{H}^{\left(N_{r}-1,0\right)} \mathbf{W}_{t}^{(0)} \\
\vdots \\
\mathbf{H}^{\left(N_{r}-1, M-1\right)} \mathbf{W}_{t}^{(M-1)}
\end{array}\right], \quad \mathbf{N}=\left[\begin{array}{c}
\mathbf{N}^{(0,0)} \\
\vdots \\
\mathbf{N}^{(0, M-1)} \\
\vdots \\
\vdots \\
\mathbf{N}^{\left(N_{r}-1,0\right)} \\
\vdots \\
\mathbf{N}^{\left(N_{r}-1, M-1\right)}
\end{array}\right] .
$$

Using (12), (8) can be rewritten as

$$
\hat{\mathbf{D}}^{(i)}=\mathbf{W}_{r}^{(i)} \mathbf{R}-\sqrt{\frac{2 E_{s}}{T_{s}}}\left\{\mathbf{W}_{r}^{(i)} \overline{\mathbf{H}}-\mathbf{I}\right\} \widetilde{\mathbf{D}}^{(i-1)},
$$

where

$$
\begin{aligned}
& \mathbf{W}_{r}^{(i)} \\
& =\left[\mathbf{W}_{r}^{(0,0, i)} \cdots \mathbf{W}_{r}^{(0, M-1, i)} \cdots \mathbf{W}_{r}^{\left(N_{r}-1,0, i\right)} \cdots \mathbf{W}_{r}^{\left(N_{r}-1, M-1, i\right)}\right] .
\end{aligned}
$$




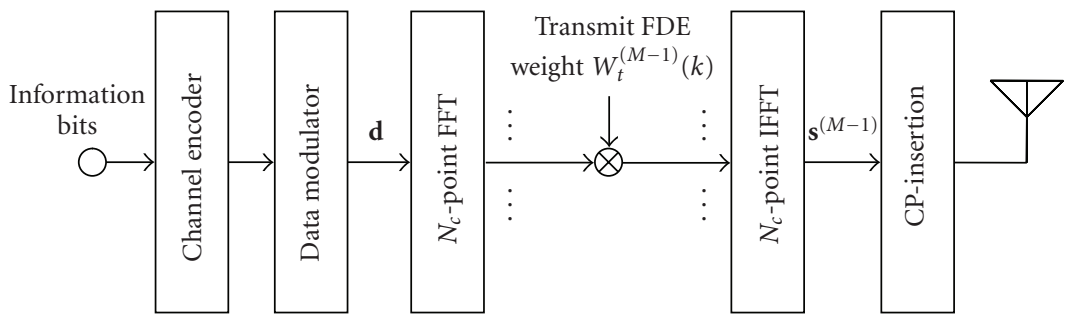

(a) Transmitter.

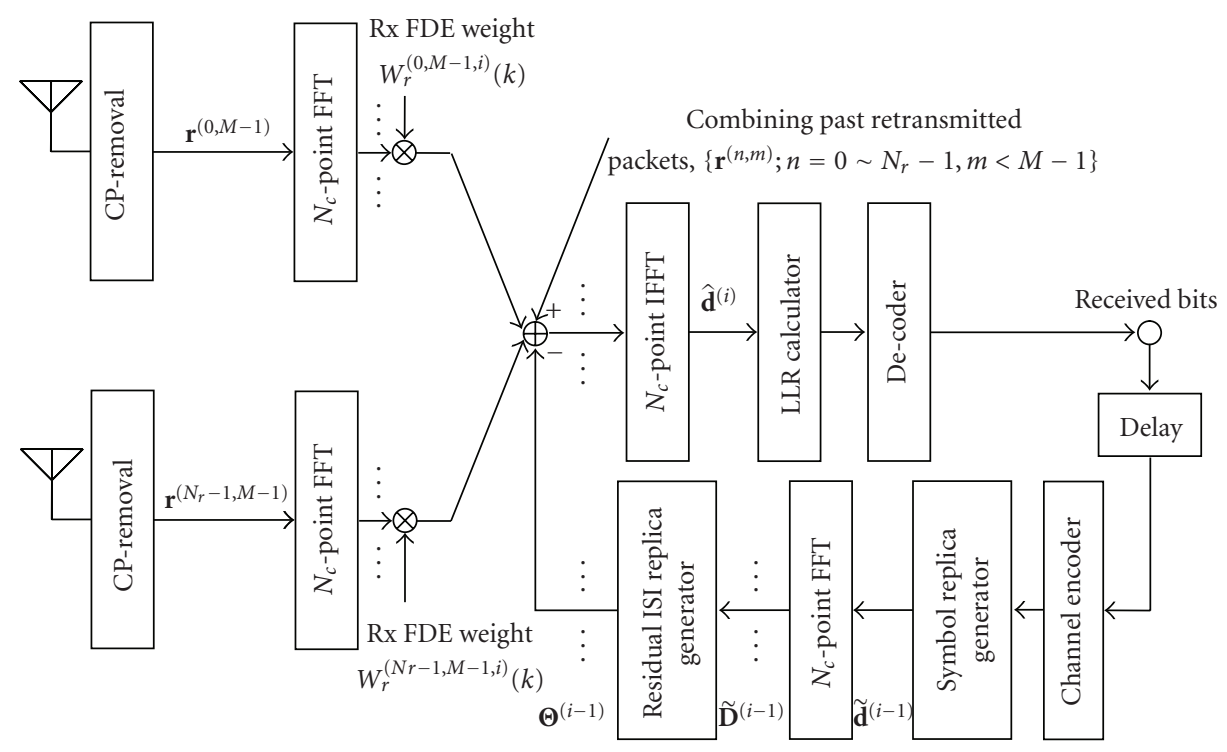

(b) Receiver.

FIgURE 2: Transmitter/receiver structure.

The error vector $\mathbf{e}^{(i)}=\left[e^{(i)}(0), \ldots, e^{(i)}(t), \ldots, e^{(i)}\left(N_{c}-\right.\right.$ 1) $]^{T}$ between $\mathbf{d}$ and $\widehat{\mathbf{d}}^{(i)}=\mathbf{F}^{H} \widehat{\mathbf{D}}^{(i)}$ at the ith iteration stage is de-fined as

$$
\begin{aligned}
\mathbf{e}^{(i)} & =\frac{\mathbf{d}-\widehat{\mathbf{d}}^{(i)}}{\sqrt{2 E_{s} / T_{s}}} \\
& =\mathbf{F}^{H}\left\{\mathbf{W}_{r}^{(i)} \overline{\mathbf{H}}-\mathbf{I}\right\}\left\{\mathbf{D}-\widetilde{\mathbf{D}}^{(i-1)}\right\}+\frac{\mathbf{F}^{H} \mathbf{W}_{r}^{(i)} \mathbf{N}}{\sqrt{2 E_{s} / T_{s}}} .
\end{aligned}
$$

The total MSE $e^{(i)}=\operatorname{tr}\left[E\left(\mathbf{e}^{(i)}\left\{\mathbf{e}^{(i)}\right\}^{H}\right)\right]$ is given as

$$
\begin{aligned}
e^{(i)}= & \rho^{(i-1)} \cdot \operatorname{tr}\left[\left\{\mathbf{W}_{r}^{(i)} \overline{\mathbf{H}}-\mathbf{I}\right\}\left\{\mathbf{W}_{r}^{(i)} \overline{\mathbf{H}}-\mathbf{I}\right\}^{H}\right] \\
& +\gamma^{-1} \cdot \operatorname{tr}\left[\mathbf{W}_{r}^{(i)}\left\{\mathbf{W}_{r}^{(i)}\right\}^{H}\right],
\end{aligned}
$$

where $\gamma$ is the average symbol energy-to-noise power spectrum density ratio $\left(E_{s} / N_{0}\right)$ and $\operatorname{tr}(\cdot)$ denotes the trace of the matrix. $\rho^{(i-1)}$ represents the residual ISI power estimated by the receiver after the $i$ th iteration of iterative Rx MMSE-FDE and ISIC and is given by [8-10]

$$
\rho^{(i-1)} \cdot \mathbf{I}=E\left[\left\{\mathbf{D}-\widetilde{\mathbf{D}}^{(i-1)}\right\}\left\{\mathbf{D}-\widetilde{\mathbf{D}}^{(i-1)}\right\}^{H}\right] .
$$

The MMSE solution of $\mathbf{W}_{r}^{(i)}$ is the one that minimizes $e^{(i)}$. From (17), we obtain

$$
\mathbf{W}_{r}^{(i)}=\overline{\mathbf{H}}^{H}\left\{\overline{\mathbf{H H}}^{H}+\left(\gamma \rho^{(i-1)}\right)^{-1} \cdot \mathbf{I}\right\}^{-1} .
$$

Using the matrix inversion lemma [1], the MMSE solution of $\mathbf{W}_{r}^{(n, m, i)}$ on the $n$th receive antenna for the $m$ th retransmission can be derived as (Appendix A)

$$
\begin{aligned}
\mathbf{W}_{r}^{(n, m, i)}= & \left\{\sum_{n^{\prime}=0}^{N_{r}-1} \sum_{m^{\prime}=0}^{M-1} \mathbf{H}^{\left(n^{\prime}, m^{\prime}\right)} \mathbf{W}_{t}^{\left(m^{\prime}\right)}\left\{\mathbf{H}^{\left(n^{\prime}, m^{\prime}\right)} \mathbf{W}_{t}^{\left(m^{\prime}\right)}\right\}^{H}\right. \\
& \left.+\left(\gamma \rho^{(i-1)}\right)^{-1} \mathbf{I}\right\}^{-1} \times\left\{\mathbf{H}^{(n, m)} \mathbf{W}_{t}^{(m)}\right\}^{H} .
\end{aligned}
$$

3.2. Transmit MMSE-FDE Weight. The transmitter predicts the degree of the residual ISI after the iteration process in the receiver. The predicted value of $\rho^{(I-1)}$ after the receiver performs the iterative Rx MMSE-FDE and ISIC is denoted by $\rho_{t x}$. The transmitter believes that the residual ISI power is reduced by a factor of $\rho_{t x}$ by a series of receive MMSE-FDE, antenna diversity combining, packet combining, and ISIC is carried out in an iterative manner at the receiver. The error 
vector $\mathbf{e}^{(M-1, t x)}$ which corresponds to (16) can be given, based on the prediction of the degree of residual ISI, as

$$
\mathbf{e}^{(M-1, t x)}=\sqrt{\rho_{t x}} \cdot \mathbf{F}^{H}\left\{\mathbf{W}_{r}^{(t x)} \overline{\mathbf{H}}-\mathbf{I}\right\} \mathbf{D}+\frac{\mathbf{F}^{H} \mathbf{W}_{r}^{(t x)} \mathbf{N}}{\sqrt{2 E_{s} / T_{s}}},
$$

where $\mathbf{W}_{r}{ }^{(t x)}$ represents the virtual $N_{c} \times M N_{r} N_{c}$ receive FDE weight matrix similar to (15). Similar to the derivation of (18), the MMSE solution that minimizes $e^{(M-1, t x)}=\operatorname{tr}\left[E\left(\mathbf{e}^{(M-1, t x)}\left\{\mathbf{e}^{(M-1, t x)}\right\}^{H}\right)\right]$ can be derived as

$$
\mathbf{W}_{r}^{(t x)}=\overline{\mathbf{H}}^{H}\left\{\overline{\mathbf{H H}}^{H}+\left(\gamma \rho_{t x}\right)^{-1} \cdot \mathbf{I}\right\}^{-1} .
$$

Substituting (22) into (21), we obtain

$$
\begin{aligned}
& e^{(M-1, t x)} \\
& =\gamma^{-1} \cdot \operatorname{tr}\left[\left\{\overline{\mathbf{H}}^{H} \overline{\mathbf{H}}+\left(\gamma \rho_{t x}\right)^{-1}\right\}^{-1}\right] \\
& =\sum_{k=0}^{N_{c}-1} \frac{\rho_{t x}}{\gamma \rho_{t x} \sum_{m=0}^{M-1}\left|W_{t}^{(m)}(k)\right|^{2} \sum_{n=0}^{N_{r}-1}\left|H^{(n, m)}(k)\right|^{2}+1} .
\end{aligned}
$$

The MMSE solution $\mathbf{W}_{t}^{(M-1)}$ can be derived using the Lagrange multiplier method [1] under the transmit power constraint $\operatorname{tr}\left[\mathbf{W}_{t}^{(M-1)}\left\{\mathbf{W}_{t}^{(M-1)}\right\}^{H}\right]=N_{c}$ for the given $\left\{\mathbf{H}^{(m)}\right.$; $m=0 \sim M-1\}$ and $\left\{\mathbf{W}_{t}^{(m)} ; m<M-1\right\}$ as (derivation is shown in Appendix B)

$$
\begin{aligned}
& \mathbf{W}_{t}^{(M-1)} \\
& \quad=\operatorname{diag}\left\{W_{t}^{(M-1)}(0), \ldots, W_{t}^{(M-1)}(k), \ldots, W_{t}^{(M-1)}\left(N_{c}-1\right)\right\},
\end{aligned}
$$

with

$$
\begin{aligned}
\left|W_{t}^{(M-1)}(k)\right|^{2} & \\
= & \max \left[\left\{\frac{1}{\mu} \sqrt{\frac{\gamma^{-1}}{\sum_{n=0}^{N_{r}-1}\left|H^{(n, M-1)}(k)\right|^{2}}}\right.\right. \\
& -\frac{\gamma^{-1}}{\rho_{t x} \sum_{n=0}^{N_{r}-1}\left|H^{(n, M-1)}(k)\right|^{2}} \\
& \left.\left.-\frac{\sum_{m<M-1}\left|W_{t}^{(m)}(k)\right|^{2} \sum_{n=0}^{N_{r}-1}\left|H^{(n, m)}(k)\right|^{2}}{\sum_{n=0}^{N_{r}-1}\left|H^{(n, M-1)}(k)\right|^{2}}\right\}, 0\right],
\end{aligned}
$$

where $\mu$ is chosen so as to satisfy $\operatorname{tr}\left[\mathbf{W}_{t}^{(M-1)} \mathbf{W}_{t}^{(M-1) H}\right]=N_{c}$.

As mentioned earlier, $\rho_{t x}$ represents the predicted residual ISI power. $\rho^{(I-1)}$ represents the residual ISI power estimated by the receiver after the $I$ th iteration in the iterative Rx MMSE-FDE and ISIC. If the transmitter believes that the receiver can perfectly cancel the residual ISI, $\rho_{t x}$ in (25) should be 0 . When $\rho_{t x}$ approaches zero, the second term of (25) becomes very large and therefore, all the transmit FDE weights are replaced by zero's except those at frequencies having the highest channel gain. In this case, transmit FDE will severely distort the transmit signal spectrum and produce a large amount of residual ISI at the receiver, thereby significantly degrading the performance. On the other hand, if the transmitter believes that the receiver cannot cancel the residual ISI at all (or when the iterative ISIC is not employed), $\rho_{t x}$ in (25) should be 1 . In this case, since the transmitter predicts no ISI suppression at the receiver, the transmit FDE tries, before the packet transmission, to reduce the frequency-selectivity of the equivalent channel seen by the receiver, thereby reducing the frequency-diversity gain. As a consequence, the optimum $\rho_{t x}$ does exist and it depends on the channel condition, data modulation, coding rate, average transmit $E_{s} / N_{0}$, and the number of iterations. However, it is quite difficult, if not impossible, to find the optimum $\rho_{t x}$ for the given instantaneous conditions. In this paper, we found the value of $\rho_{t x}$ that maximizes the throughput by the preliminary computer simulation at each average transmit $E_{s} / N_{0}$ for the given channel power delay profile, data modulation, coding rate, and the number of iterations in the receiver.

\section{Performance Evaluation}

The performance of SC-HARQ using the joint iterative $\mathrm{Tx} / \mathrm{Rx}$ MMSE-FDE and ISIC is evaluated by computer simulation assuming $N_{c}=256, \mathrm{CP}$ length of $N_{g}=32$. Both QPSK and 16 QAM are assumed for data modulation. The channel is assumed to be an $L=16$-path frequencyselective block Rayleigh fading channel having exponential power delay profile with decay factor $\beta$. A turbo encoder with the original coding rate $1 / 3$ using two $(13,15)$ recursive systematic convolutional encoders [19] is used. A 2048 bit length codeword with the coding rate $R=1 / 2$ is generated by puncturing the parity bit sequences. The decoder consists of two log-MAP decoders. An error-free ACK/NACK transmission is assumed.

Two different channel cases are considered.

Channel case 1: Independent channel for each retransmission.

Channel case 2: Identical channel for each retransmission.

4.1. Channel and Tx FDE Weight for the Initial and First Retransmission $(M=2)$. In this subsection, we discuss the transmit MMSE-FDE weight for the case when the same packet has been transmitted 2 times $(M=2) . N_{r}=1$ and uniform power delay profile $(\beta=0 \mathrm{~dB})$ are assumed. Also $\rho_{t x}=0.8$ is assumed as an example. The average transmit $E_{s} / N_{0}$ is set to $5 \mathrm{~dB}$.

Figure 3(a) shows the channel gain and the transmit MMSE-FDE weight for the channel case 1. The magnitude of the transmit MMSE-FDE weight $W_{t}^{(m=0)}(k)$ is plotted in Figure 3(a). Also plotted is the amplitude of the channel gain 


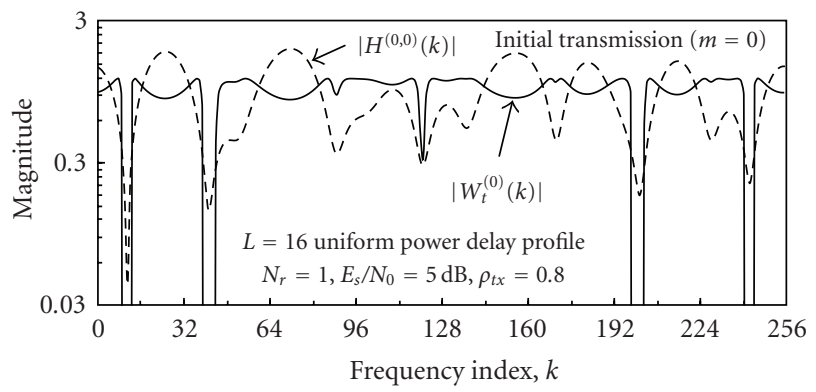

(a) $m=0$ (initial transmission).

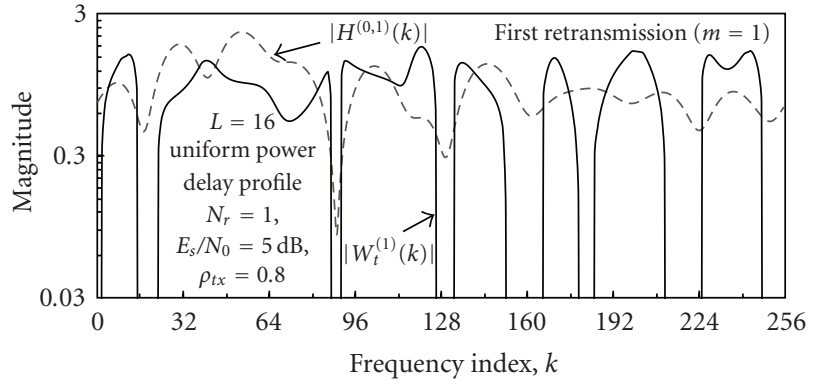

(b) $m=1$ (first retransmission).

Figure 3: Channel transfer function and Tx MMSE-FDE weight for channel case 1.

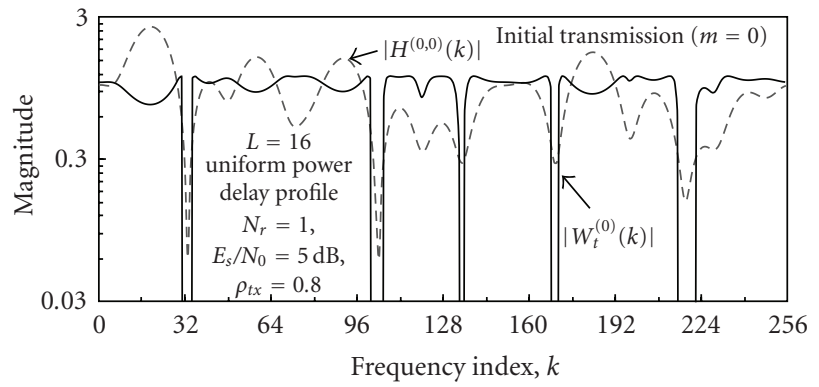

(a) $m=0$ (initial transmission).

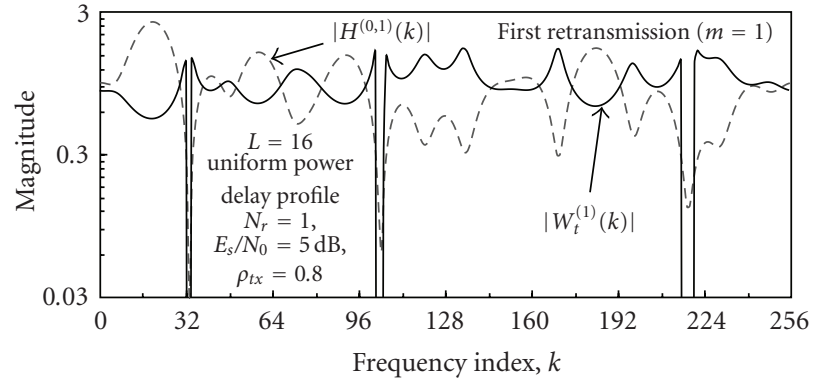

(b) $m=1$ (first retransmission).

Figure 4: Channel transfer function and Tx MMSE-FDE weight for channel case 2.

$H^{(m=0)}(k)$. The value of $\left|W_{t}^{(m=0)}(k)\right|$ is identical to the one we have previously proposed in [11] (note that in [11], the packet combining is not taken into account for computing the transmit MMSE-FDE weight). It can be seen from Figure 3 (a) that at the initial transmission $(m=0)$, the transmit MMSE-FDE weight allocates more power to the frequencies having a good condition and no power $\left(\left|W_{t}^{(m=0)}(k)\right|=0\right)$ at some frequencies having a bad condition. The magnitude of the transmit MMSE-FDE weight $W_{t}^{(m=1)}(k)$ for the first retransmission is plotted in Figure 3(b). It can be seen that $\left|W_{t}^{(m=1)}(k)\right|=0$ occurs more frequently. For the first retransmission $(m=1)$, the transmit MMSE-FDE weight $\left|W_{t}^{(m=1)}(k)\right|$ more likely has large magnitude at the frequencies with $\left|W_{t}^{(m=0)}(k)\right|=0$ so as to make the equivalent channel seen after the packet combining nearly flat. Every time a new retransmission is to be made, the new transmit MMSE-FDE weight allocates more power to the frequencies which have not been allocated enough power at the previous retransmissions; in other words, the transmit MMSE-FDE weight tends to become zero on a frequency which has been allocated enough power already at previous retransmissions. For example, in Figure 3(a), the value of $\left|W_{t}{ }^{(m=0)}(k=12)\right|$ for the initial transmission is zero. However, the value of $\left|W_{t}^{(m=1)}(k=12)\right|$ for the first retransmission becomes very high so as to rectify the variations in the equivalent channel after packet combining over the signal bandwidth.

Figure 4 shows the channel gain and the transmit FDE weight for the channel case 2 . Since the channel gain stays the same for the initial transmission and the first retransmission, no time diversity gain is obtained. However, the transmit FDE weight for the retransmission changes from the initial transmission and therefore, the channel amplitude variations in the frequency-domain can be slightly reduced after the packet combining (see Figures 4(a) and $4(\mathrm{~b}))$.

4.2. Average Packet Error Rate (PER) Performance. Figure 5 shows the achievable average PER performance, achievable by our proposed joint iterative Tx/Rx MMSE-FDE and ISIC as a function of the average transmit $E_{s} / N_{0}$ with the number of iterations, $I$, as a parameter for $16 \mathrm{QAM}$, the channel case 1 with the decay factor $\beta=0 \mathrm{~dB}$ (uniform power delay profile), and $N_{r}=1$ (no receive diversity). The same packet is assumed to have been transmitted $M$ times. The PER performance achieved by the conventional scheme (iterative Rx MMSE-FDE and ISIC without transmit FDE proposed in $[9,10])$ is also plotted for comparison. It can be seen that as $I$ increases, PER performance of both schemes improves. In comparison to the conventional scheme, the proposed scheme always provides better PER performance for the same value of $I$. When $M=1$, the performance difference between the proposed and conventional schemes becomes narrower when $I \geq 6$ (see Figure 5(a)); the reduction in the required $E_{s} / N_{0}$ for achieving PER $=0.1$ is only about $0.5 \mathrm{~dB}$ from the conventional scheme. On the other hand, when $M=3$, the performance difference becomes wider as $I$ increases thanks to the packet combining gain (see Figure 5(b)); the proposed 

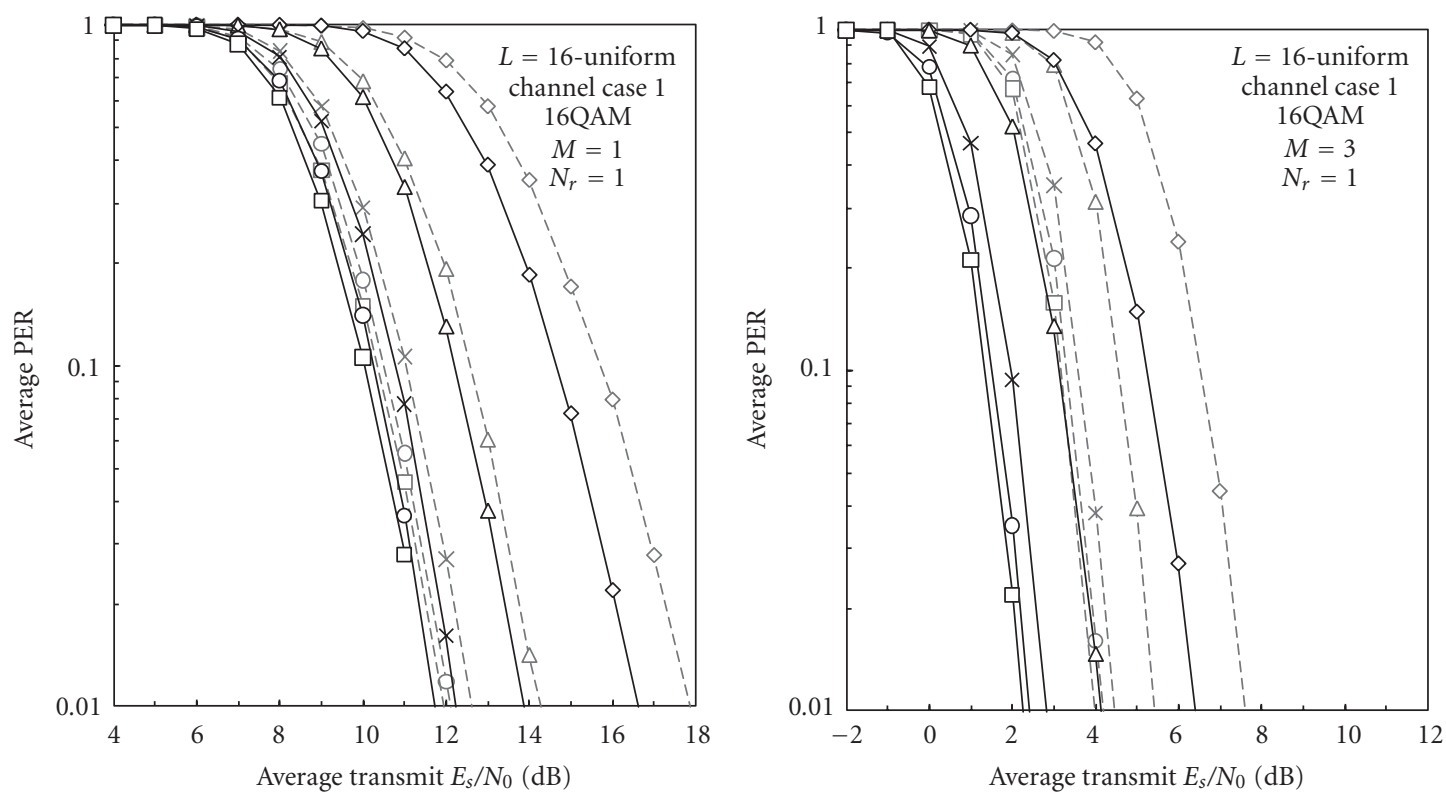

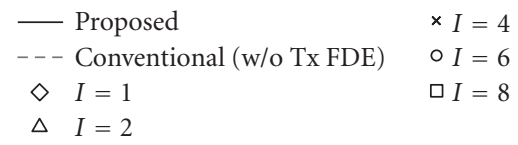

(a) $M=1$.

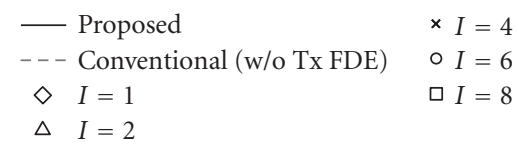

(b) $M=3$.

Figure 5: Average PER performance.

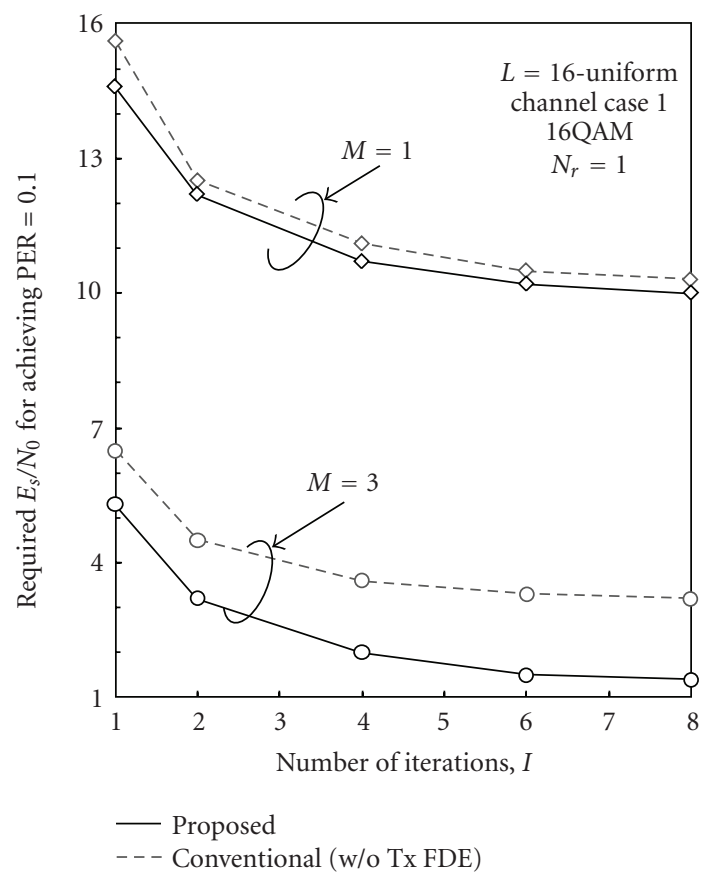

(a) $N_{r}=1$.

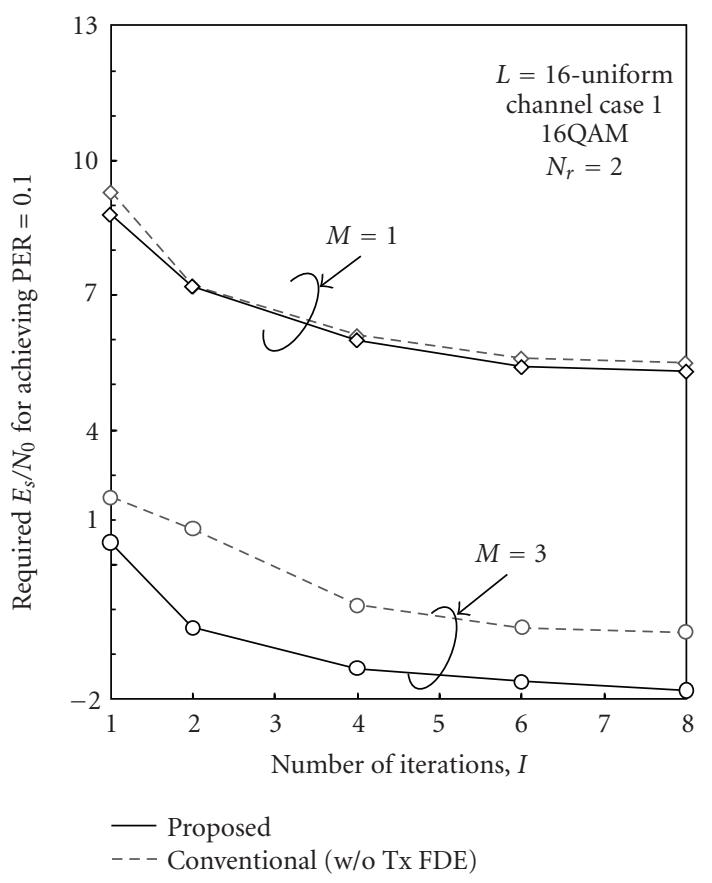

(b) $N_{r}=2$.

FIGURE 6: Impact of the number of iterations, I, on required $E_{s} / N_{0}$ for achieving PER $=0.1$. 


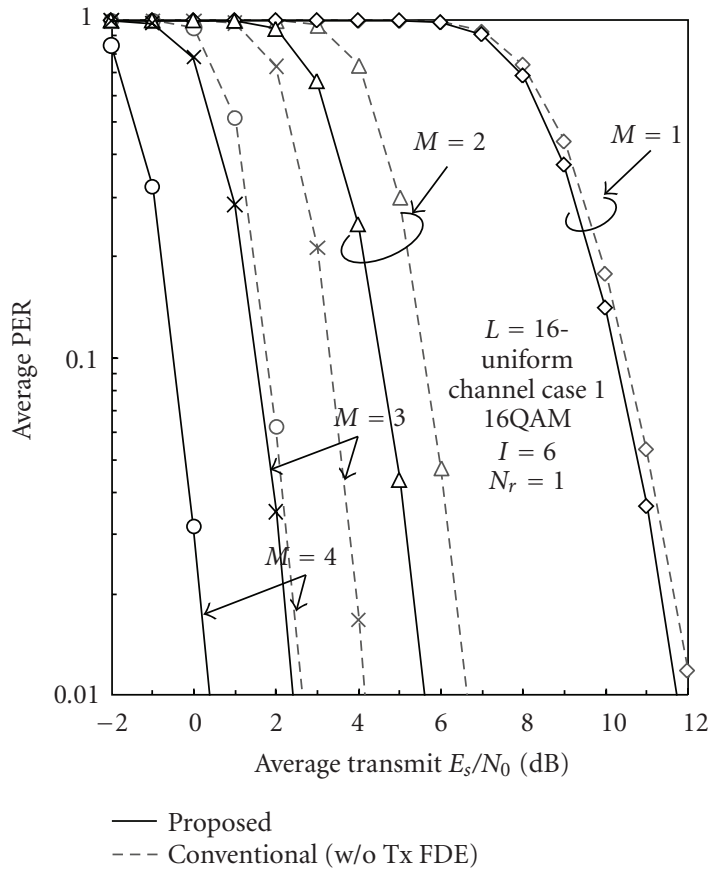

(a) $N_{r}=1$.

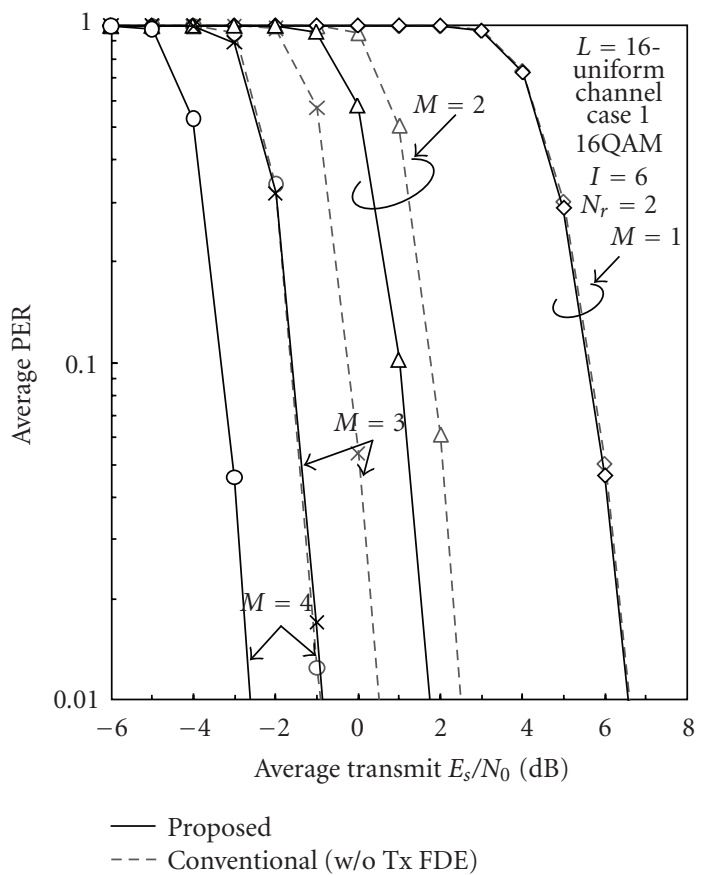

(b) $N_{r}=2$.

Figure 7: Average PER performance when $M=1,2,3$, and 4.

scheme with $I=6$ can reduce the required $E_{s} / N_{0}$ by as much as about $2.0 \mathrm{~dB}$.

4.3. Impact of the Number of Iterations on the Required $E_{s} / N_{0}$. To discuss the impact of the number of iterations, $I$, on the performance, we plot the required $E_{s} / N_{0}$ for achieving PER $=0.1$ in Figure 6 as a function of $I$ for 16 QAM and the channel case 1 with the decay factor $\beta=$ $0 \mathrm{~dB}$ (uniform power delay profile). It can be seen from Figure 6 that the packet combining significantly reduces the required $E_{s} / N_{0}$. When $M=3$, the proposed scheme provides larger reduction in the required $E_{s} / N_{0}$ than the conventional scheme since the proposed scheme achieves higher packet combining gain due to the use of transmit MMSE-FDE. When $M=1$, only a slight difference in the required $E_{s} / N_{0}$ between the proposed and conventional schemes is seen, especially for the case of $N_{r}=2$ (receive antenna diversity). This is because the use of antenna diversity reception tries to flatten the equivalent channel transfer function seen by the transmitter. The transmit FDE does not alter the spectrum shape for the flat channel case at all and therefore, the proposed scheme can reduce the required $E_{s} / N_{0}$ only slightly.

Figure 6 also shows that the required $E_{s} / N_{0}$ reduction is almost saturated when $I \geq 6$. Therefore, we will use $I=6$ in the following evaluations.

4.4. Impact of the Number of the Packet Retransmissions on Average PER Performance. The impact of the number of packet retransmissions, $M$, on the average PER performance is discussed below. The average PER performance of the proposed scheme is plotted in Figure 7 as a function of average transmit $E_{s} / N_{0}$ with $M$ as a parameter. For comparison, the PER performance of the conventional scheme is also plotted. It can be seen from Figure 7 that as $M$ increases, the proposed scheme provides much better PER performance than the conventional scheme thanks to the use of transmit MMSE-FDE for each retransmission. The reduction in the required $E_{s} / N_{0}$ for achieving $\mathrm{PER}=0.1$ from the conventional scheme is about $0.5 \mathrm{~dB}, 1.0 \mathrm{~dB}, 2.0 \mathrm{~dB}$, and $2.7 \mathrm{~dB}$ when $N_{r}=1$ (no receive antenna diversity) and is about $0.1 \mathrm{~dB}, 0.7 \mathrm{~dB}, 1.3 \mathrm{~dB}$, and $2.0 \mathrm{~dB}$ when $N_{r}=2$ (receive antenna diversity) for $M=1,2,3$, and 4 , respectively. Reducing the required $E_{s} / N_{0}$ leads to achieving the higher throughput.

4.5. Throughput Performance. The achievable throughput performance by the proposed scheme is plotted as a function of average transmit $E_{s} / N_{0}$ in Figure 8 for QPSK and in Figure 9 for 16 QAM. Two cases of the decay factor $\beta$ of the power delay profile are considered. The throughput performance achievable by the conventional scheme is also plotted for comparison. It can be seen from Figures 8 and 9 that the proposed scheme always provides higher throughput than the conventional scheme except for the channel case 2 when 16 QAM is used (16 QAM is more sensitive than QPSK to the residual ISI after packet combining and ISIC).

At first, the case of $\beta=0 \mathrm{~dB}$ (equivalent to the uniform power delay profile) is discussed. In this case, larger frequency-diversity gain is obtained and thus, the 


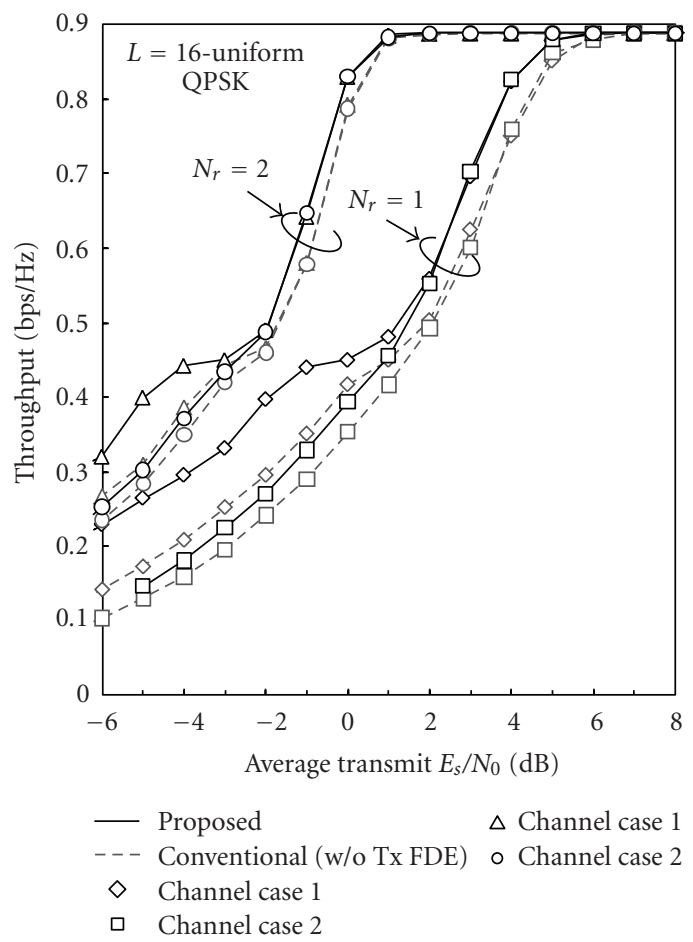

(a) $\beta=0 \mathrm{~dB}$ (uniform power delay profile).

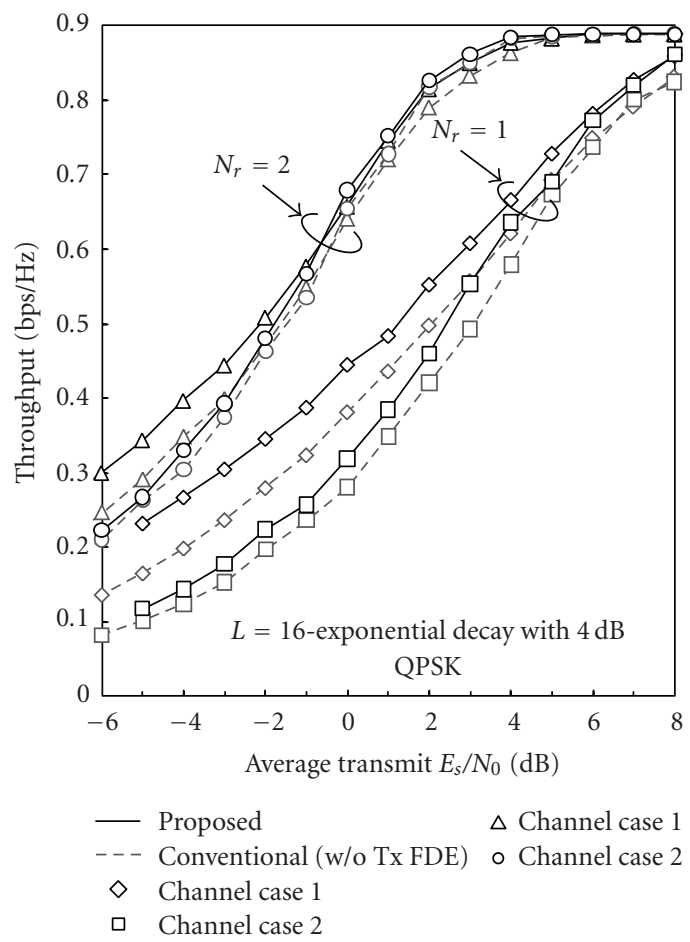

(b) $\beta=4 \mathrm{~dB}$ (exponentially decaying power delay profile).

FIgURE 8: HARQ throughput with QPSK.

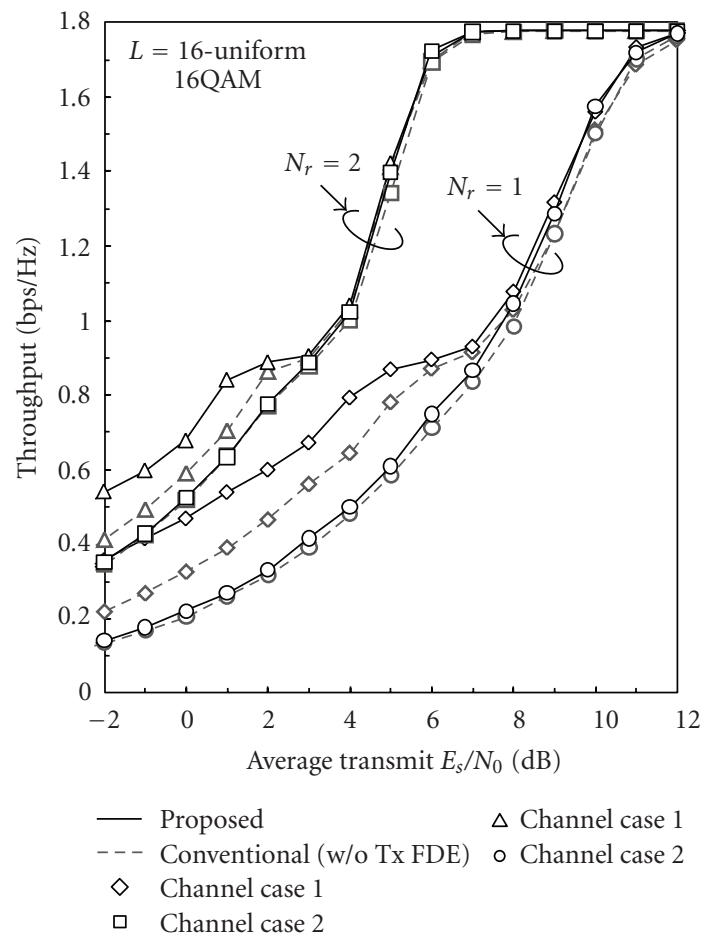

(a) $\beta=0 \mathrm{~dB}$ (uniform power delay profile).

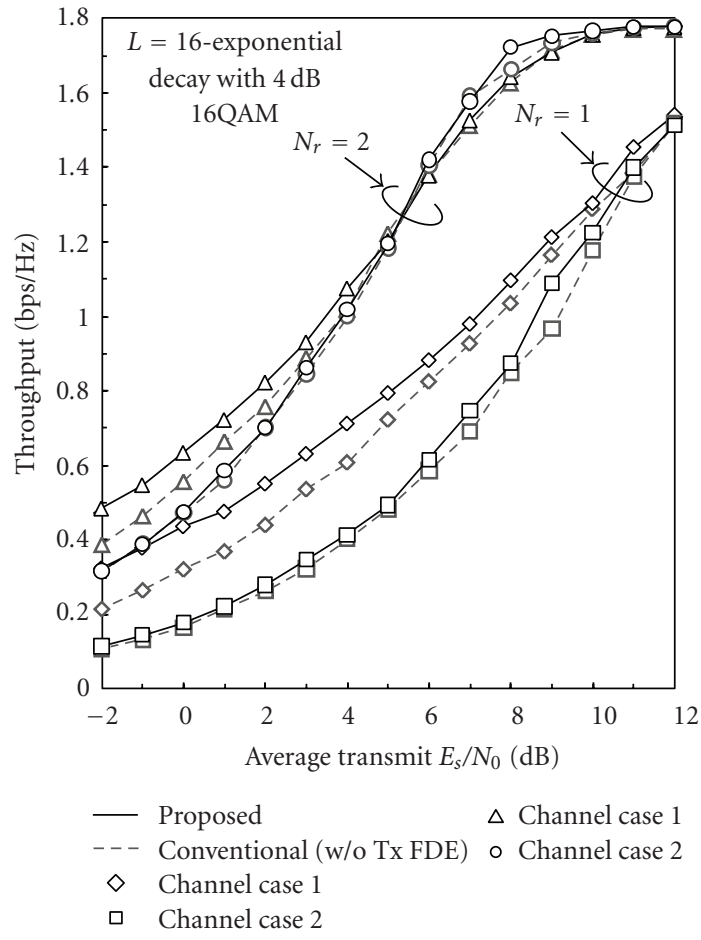

(b) $\beta=4 \mathrm{~dB}$ (exponentially decaying power delay profile).

FIgURE 9: HARQ throughput with 16 QAM. 


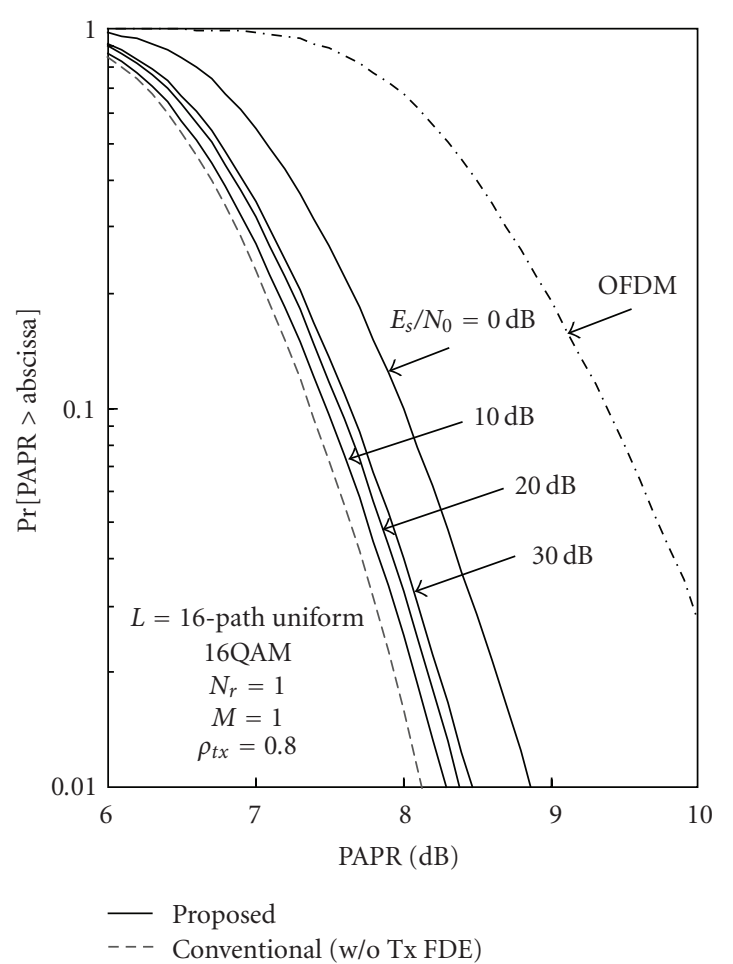

Figure 10: PAPR distribution with the average transmit $E_{s} / N_{0}$ as a parameter.

initial packet transmission is likely successful in a high average transmit $E_{s} / N_{0}$ region. As the average transmit $E_{s} / N_{0}$ decreases, the packet error probability at the initial transmission increases and consequently, the packet retransmission is likely requested. For the channel case 1 (independent channel for each retransmission), time-diversity gain can be achieved through the packet combining. When $N_{r}=$ 1 , most likely the packet can be correctly received after the first retransmission at around the average transmit $E_{s} / N_{0}=0 \mathrm{~dB}(6 \mathrm{~dB})$ as shown in Figure 8(a) (Figure 9(a)); the flat throughput region is observed with the proposed scheme. Therefore, the throughput difference between the proposed and conventional schemes gets larger as the average transmit $E_{s} / N_{0}$ decreases. On the other hand, for the channel case 2 (identical channel for all retransmissions), the timediversity gain cannot be obtained through packet combining. Therefore, the throughput difference is kept at almost the same irrespective of the average transmit $E_{s} / N_{0}$. When $N_{r}=2$ (receive antenna diversity), the residual ISI can be significantly reduced by the antenna diversity reception. Therefore, the performance improvement achievable by the proposed scheme over the conventional scheme becomes negligible in a high $E_{s} / N_{0}$ region. However, as already discussed in Figure 7(b), the proposed scheme can still obtain larger packet combining gain even when $N_{r}=$ 2 , thereby achieving higher throughput in a low $E_{s} / N_{0}$ region.

Next, the case of $\beta=4 \mathrm{~dB}$ is discussed. In this case, the proposed scheme provides the performance improvement similar to the case of $\beta=0 \mathrm{~dB}$; however, the flat throughput

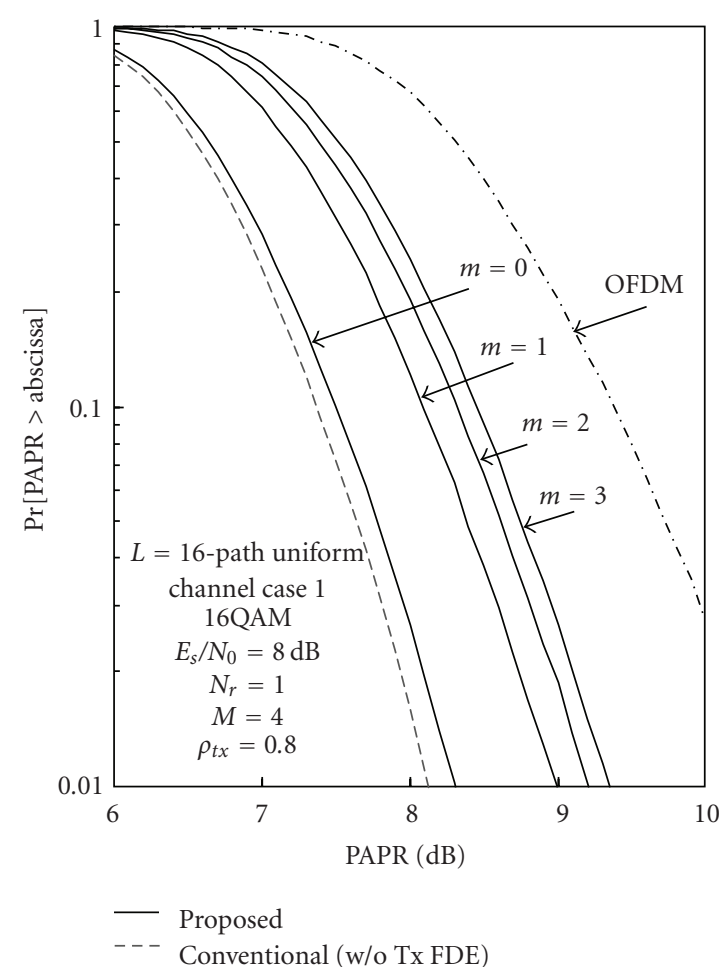

FIgURE 11: PAPR distribution with $\mathrm{m}$ as a parameter for $M=4$.

region is not observed. This is because, due to the lower frequency-diversity gain, the 1st retransmission is not always sufficient for correct packet reception.

4.6. Peak-to-Average Power Ratio (PAPR) Issue. The complementary cumulative distribution function (CCDF) of peakto-average power ratio (PAPR) with our proposed scheme is plotted in Figure 10 for 16 QAM with the average transmit $E_{s} / N_{0}$ as a parameter when $M=1$ (no ARQ is used). It is assumed that $\rho_{t x}=0.8, \beta=0 \mathrm{~dB}$ (equivalent to uniform power delay profile), and $N_{r}=1$ (no antenna diversity). The PAPR is measured by 4 -time oversampling of the transmit SC signals after transmit FDE. The CCDFs of PAPR for the conventional scheme (equivalent to SC without transmit FDE) and orthogonal frequency division multiplexing (OFDM) are also plotted for comparison. The PAPR distribution of the proposed scheme depends on the average transmit $E_{s} / N_{0}$. It can be seen that when the average transmit $E_{s} / N_{0}=0 \mathrm{~dB}$, the PAPR level at which the measured PAPRs exceed with $10 \%$ probability (this is called the PAPR 10\% level in this paper) increases by about $0.7 \mathrm{~dB}$ compared to SC without transmit FDE. As the average transmit $E_{s} / N_{0}$ increases, the PAPR $10 \%$ level reduces. When the average transmit $E_{s} / N_{0}=30 \mathrm{~dB}$, the PAPR $10 \%$ level with our proposed scheme is about $0.3 \mathrm{~dB}$ higher than that with the conventional scheme. However, the PAPR 10\% level of the proposed scheme is lower than that of OFDM signals.

The PAPR distribution of the proposed scheme depends on the number of retransmissions, $m$, for the fixed value of 
$M$. Figure 11 plots the PAPR distribution for the different values of $m$ when $M=4$. The channel case 1 and the average transmit $E_{s} / N_{0}=8 \mathrm{~dB}$ are assumed. The PAPR 10\% levels for the conventional scheme and OFDM are also plotted for comparison. It can be seen that the PAPR 10\% level increases as $m$ increases. The increase in the PAPR 10\% level from the case of $m=0$ (initial transmission) is about $0.6 \mathrm{~dB}, 0.9 \mathrm{~dB}$, and $1.1 \mathrm{~dB}$ when $m=1,2$, and 3 , respectively. However, the PAPR $10 \%$ level of the proposed scheme is still lower than that of OFDM signals irrespective of $m$.

As seen from Figures 10 and 11, the introduction of transmit FDE increases the PAPR level of SC signals. How the PAPR can be reduced is left as an important future study topic.

\section{Conclusion}

In this paper, we proposed the joint iterative $\mathrm{Tx} / \mathrm{Rx}$ MMSEFDE and ISIC suitable for SC-HARQ with CC. Every time the same packet is received, the receive MMSE-FDE weights are updated for combining all the retransmitted packets. The transmit MMSE-FDE weight is determined based on the prediction of the degree of residual ISI after ISIC in a receiver. We derived a set of transmit and receive MMSE-FDE weights. We showed by computer simulation that the proposed scheme achieves better PER performance than the conventional iterative Rx MMSE-FDE and ISIC. When 16QAM is used, the proposed scheme with $M=$ 3 can reduce the required $E_{s} / N_{0}$ for achieving $\mathrm{PER}=$ 0.1 by about $2.4 \mathrm{~dB}$ from the conventional scheme with $M=3$, thanks to the packet combining gain. Also shown was the throughput performance improvement. The proposed scheme was shown to be more effective for the retransmission.

In this paper, we assume perfect knowledge of CSI. How the imperfect CSI affects the PER and throughput performances achievable by the proposed joint iterative $\mathrm{Tx} / \mathrm{Rx}$ MMSE-FDE and ISIC is left as an important future study topic. (R3-1)The introduction of transmit FDE increases the PAPR level of SC signals. PAPR reduction techniques [2023] proposed for OFDM may be applied to the proposed scheme. This is left as another important future study topic.

\section{Appendices}

\section{A. Derivation of $(20)$}

Using the matrix inversion lemma, we have

$$
\begin{aligned}
& \left\{\overline{\mathbf{H H}}^{H}+\left(\gamma \rho^{(i-1)}\right)^{-1} \cdot \mathbf{I}\right\}^{-1} \\
& =\gamma \rho^{(i-1)} \cdot \mathbf{I}-\gamma \rho^{(i-1)} \overline{\mathbf{H}}\left\{\overline{\mathbf{H}}^{H}\left(\gamma \rho^{(i-1)}\right) \overline{\mathbf{H}}+\mathbf{I}\right\}^{-1} \overline{\mathbf{H}}^{H}\left(\gamma \rho^{(i-1)}\right) \\
& =\gamma \rho^{(i-1)}\left[\mathbf{I}-\overline{\mathbf{H}}\left\{\overline{\mathbf{H}}^{H} \overline{\mathbf{H}}+\left(\gamma \rho^{(i-1)}\right)^{-1} \mathbf{I}\right\}^{-1} \overline{\mathbf{H}}^{H}\right]
\end{aligned}
$$

and therefore, (19) can be rewritten as

$$
\begin{aligned}
\mathbf{W}_{r}^{(i)} & =\overline{\mathbf{H}}^{H}\left\{\overline{\mathbf{H H}}^{H}+\left(\gamma \rho^{(i-1)}\right)^{-1} \cdot \mathbf{I}\right\}^{-1} \\
& =\gamma \rho^{(i-1)} \overline{\mathbf{H}}^{H}\left[\mathbf{I}-\overline{\mathbf{H}}\left\{\overline{\mathbf{H}}^{H} \overline{\mathbf{H}}+\left(\gamma \rho^{(i-1)}\right)^{-1} \mathbf{I}\right\}^{-1} \overline{\mathbf{H}}^{H}\right] \\
& =\gamma \rho^{(i-1)}\left[\mathbf{I}-\overline{\mathbf{H}}^{H} \overline{\mathbf{H}}\left\{\overline{\mathbf{H}}^{H} \overline{\mathbf{H}}+\left(\gamma \rho^{(i-1)}\right)^{-1} \mathbf{I}\right\}^{-1}\right] \overline{\mathbf{H}}^{H} \\
& =\gamma \rho^{(i-1)}\left[\left(\gamma \rho^{(i-1)}\right)^{-1}\left\{\overline{\mathbf{H}}^{H} \overline{\mathbf{H}}+\left(\gamma \rho^{(i-1)}\right)^{-1} \mathbf{I}\right\}^{-1}\right] \overline{\mathbf{H}}^{H} \\
& =\left\{\overline{\mathbf{H}}^{H} \overline{\mathbf{H}}+\left(\gamma \rho^{(i-1)}\right)^{-1} \mathbf{I}\right\}^{-1} \overline{\mathbf{H}}^{H} .
\end{aligned}
$$

Substituting $\overline{\mathbf{H}}$ of (13) into (A.2), we obtain (20).

\section{B. Derivation of $(24)$}

We want to derive the transmit MMSE-FDE weight $\left|W_{t}^{(M-1)}(k)\right|^{2}$ for the present $(M-1)$ th retransmission when the previous transmissions have used the transmit MMSE-FDE weights $\left|W_{t}^{(m)}(k)\right|^{2}, m<M-1$. The MMSE problem for minimizing (23) under the constraint of $\operatorname{tr}\left[\mathbf{W}_{t}^{(M-1)} \mathbf{W}_{t}^{(M-1) H}\right]=N_{c}$ can be rewritten as

$$
\begin{aligned}
& \min e^{(M-1, t x)} \\
& =\sum_{k=0}^{N_{c}-1} \frac{\rho_{t x}}{\gamma \rho_{t x} \sum_{m^{\prime}=0}^{M-1} G_{t}^{\left(m^{\prime}\right)}(k) \sum_{n^{\prime}=0}^{N_{r}-1}\left|H^{\left(n^{\prime}, m^{\prime}\right)}(k)\right|^{2}+1} \\
& \text { s.t. } \quad \sum_{k=0}^{N_{c}-1} G_{t}^{(M-1)}(k)=N_{c}, \quad G_{t}^{(M-1)}(k) \geq 0,
\end{aligned}
$$

where $\left\{G_{t}^{(m)}(k) ; m=0 \sim M-1, k=0 \sim N_{c}-1\right\}$ represents $\left\{\left|W_{t}^{(m)}(k)\right|^{2} ; m=0 \sim M-1, k=0 \sim N_{c}-1\right\}$ (i.e., $\left|W_{t}^{(m)}(k)\right|^{2}$ is replaced by $G_{t}^{(m)}(k)$ to transform the MMSE problem into a convex optimization problem [24]). For the given $\left\{G_{t}^{(m)}(k) ; m<M-1, k=0 \sim N_{c}-1\right\},\left\{H^{(n, m)}(k)\right.$; $\left.n=0 \sim N_{r}-1, m=0 \sim M-1, k=0 \sim N_{c}-1\right\}, \gamma$, and $\rho_{t x}$, optimal $\left\{G_{t}^{(M-1)}(k) ; k=0 \sim N_{c}-1\right\}$ can be derived. We define the cost function as

$$
\begin{aligned}
J= & \sum_{k=0}^{N_{c}-1} \frac{\rho_{t x}}{\rho_{t x} \gamma \sum_{m^{\prime}=0}^{M-1} G_{t}^{\left(m^{\prime}\right)}(k)^{2} \sum_{n^{\prime}=0}^{N_{r}-1}\left|H^{\left(n^{\prime}, m^{\prime}\right)}(k)\right|^{2}+1} \\
& -\mu \cdot\left\{\sum_{k=0}^{N_{c}-1} G_{t}^{(M-1)}(k)-N_{c}\right\}+\sum_{k=0}^{N_{c}-1} \eta_{k} \cdot\left\{-G_{t}^{(M-1)}(k)+0\right\},
\end{aligned}
$$

where $\mu$ and $\left\{\eta_{k} ; k=0 \sim N_{c}-1\right\}$ are Lagrange multipliers. The optimal $G_{t}^{(M-1)}(k)$ is denoted by $G_{t, \text { opt }}^{(M-1)}(k)$. 
The following Karush-Kuhn-Tucker (KKT) condition [24] must be satisfied:

$$
\begin{aligned}
& \left.\frac{\partial J}{\partial G_{t}^{(M-1)}(k)}\right|_{G_{t}^{(M-1)}(k)=G_{t, \mathrm{opt}}^{(M-1)}(k)}=0 \\
& -G_{t, \mathrm{opt}}^{(M-1)}(k) \leq 0 \\
& \eta_{k} \geq 0 \\
& \eta_{k} G_{t, \mathrm{opt}}^{(M-1)}(k)=0
\end{aligned}
$$

for $k=0 \sim N_{c}-1$ and

$$
\sum_{k=0}^{N_{c}-1} G_{t, \mathrm{opt}}^{(M-1)}(k)-N_{c}=0
$$

By solving (B.3) and (B.4), we obtain the MMSE solution, which is (24).

\section{References}

[1] J. G. Proakis, Digital Communications, McGraw-Hill, New York, NY, USA, 4th edition, 2001.

[2] H. Sari, G. Karam, and I. Jeanclaude, "Analysis of orthogonal frequency-division multiplexing for mobile radio applications," in Proceedings of the 44th IEEE Vehicular Technology Conference (VTC'94), vol. 3, pp. 1635-1639, June 1994.

[3] D. Falconer, S. L. Ariyavisitakul, A. Benyamin-Seeyar, and B. Eidson, "Frequency domain equalization for single-carrier broadband wireless systems," IEEE Communications Magazine, vol. 40, no. 4, pp. 58-66, 2002.

[4] F. Adachi, D. Garg, S. Takaoka, and K. Takeda, "Broadband CDMA techniques," IEEE Wireless Communications, vol. 12, no. 2, pp. 8-18, 2005.

[5] N. Benvenuto and S. Tomasin, "Iterative design and detection of a DFE in the frequency domain," IEEE Transactions on Communications, vol. 53, no. 11, pp. 1867-1875, 2005.

[6] K. Takeda, K. Ishihara, and F. Adachi, "Frequency-domain ICI cancellation with MMSE equalization for DS-CDMA downlink," IEICE Transactions on Communications, vol. E89B, no. 12, pp. 3335-3343, 2006.

[7] K. Ishihara, K. Takeda, and F. Adachi, "Frequency-domain multi-stage soft interference cancellation for DS-CDMA uplink signal transmission," IEICE Transactions on Communications, vol. E90-B, no. 5, pp. 1152-1161, 2007.

[8] R. Dinis, P. Silva, and T. Araújo, "Joint turbo equalization and cancelation of nonlinear distortion effects in MC-CDMA signals," in Proceedings of the 8th IASTED International Conference on Signal and Image Processing (SIP '06), pp. 367371, Honolulu, Hawaii, USA, August 2006.

[9] K. Takeda and F. Adachi, "HARQ throughput performance of multicode DS-CDMA with MMSE turbo equalization," in Proceedings of the 65th IEEE Vehicular Technology Conference (VTC '07), pp. 1603-1607, Dublin, Ireland, April 2007.

[10] R. Dinis, P. Carvalho, and J. Martins, "Soft combining ARQ techniques for wireless systems employing SC-FDE schemes," in Proceedings of the 17th International Conference on Computer Communications and Networks (ICCCN'08), pp. 174-178, St. Thomas, Virgin Islands, USA, August 2008.
[11] K. Takeda, H. Tomeba, and F. Adachi, "Multicode DSCDMA with joint transmit/receive frequency-domain equalization," in Proceedings of the 20th IEEE International Symposium on Personal, Indoor and Mobile Radio Communications (PIMRC'09), Tokyo, Japan, September 2009.

[12] K. Takeda and F. Adachi, "Joint iterative transmit/receive frequency-domain equalization \& ISI cancellation for broadband single-carrier block transmissions," in Proceedings of the 6th International Wireless Communications and Mobile Computing Conference (IWCMC '10), pp. 163-167, Caen, France, June-July 2010.

[13] D. Chase, "Code combining - a maximum-likelihood decoding approach for combining an arbitrary number of noisy packets," IEEE Transactions on Communications, vol. 33, no. 5, pp. 385-393, 1985.

[14] D. N. Rowitch and L. B. Milstein, "Rate compatible punctured turbo (RCPT) codes in a hybrid FEC/ARQ system," in Proceedings of the IEEE Global Telecommunications MiniConference (GLOVECOM '97), vol. 4, pp. 55-59, Phoenix, Ariz, USA, November 1997.

[15] D. Garg and F. Adachi, "Packet access using DS-CDMA with frequency-domain equalization," IEEE Journal on Selected Areas in Communications, vol. 24, no. 1, pp. 161-170, 2006.

[16] R. Esmailzadeh, M. Nakagawa, and A. Jones, "TDD-CDMA for the 4th generation of wireless communications," IEEE Wireless Communications, vol. 10, no. 4, pp. 8-15, 2003.

[17] K. Takeda and F. Adachi, "Impact of channel estimation error on the single-carrier joint Tx/Rx MMSE-FDE," in Proceedings of IEEE VTS Asia-Pacific Wireless Communications Symposium (APWCS '10), Kaosheong, Taiwan, May 2010.

[18] K. Takeda and F. Adachi, "Bit error rate analysis of DS-CDMA with joint frequency-domain equalization and antenna diversity combining," IEICE Transactions on Communications, vol. E87-B, no. 10, pp. 2991-3002, 2004.

[19] D. Raphaeli and Y. Zarai, "Combined turbo equalization and turbo decoding," in Proceedings of the IEEE Global Telecommunications Conference (GLOBECOM '97), vol. 2, pp. 639-641, November 1997.

[20] S. H. Han and J. H. Lee, "An overview of peak-to-average power ratio reduction techniques for multicarrier transmission," IEEE Wireless Communications, vol. 12, no. 2, pp. 56-65, 2005.

[21] B. S. Krongold and D. L. Jones, "PAR reduction in OFDM via active constellation extension," IEEE Transactions on Broadcasting, vol. 49, no. 3, pp. 258-268, 2003.

[22] H. Ochiai and H. Imai, "Performance analysis of deliberately clipped OFDM signals," IEEE Transactions on Communications, vol. 50, no. 1, pp. 89-101, 2002.

[23] P. Boonsrimuang, K. Mori, T. Paungma, and H. Kobayashi, "Proposal of simple PAPR reduction method for OFDM signal by using dummy sub-carriers," IEICE Transactions on Communications, vol. E91-B, no. 3, pp. 784-794, 2008.

[24] S. Boyd and L. Vandenberghe, Convex Optimization, Cambridge University Press, Cambridge, UK, 2004. 